\title{
Dynamic transpedicular stabilisation and decompression in single-level degenerative anterolisthesis and stenosis
}

\author{
Michael Payer • Smoll Nicolas • Neriman Oezkan • \\ Enrico Tessitore
}

Received: 4 June 2013 / Accepted: 20 November 2013 / Published online: 19 December 2013

(C) Springer-Verlag Wien 2013

\begin{abstract}
Background Different treatment options exist for symptomatic single-level degenerative anterolisthesis and stenosis. While simple micro-decompression has been advocated lately, most authors recommend posterior decompression with fusion. In recent years, decompression and dynamic transpedicular stabilisation has been introduced for this indication. The aim of this study was to evaluate the safety and efficacy of decompression and dynamic transpedicular stabilisation with the Dynesys ${ }^{\circledR}$ system in single-level degenerative anterolisthesis and stenosis.

Methods Thirty consecutive patients with symptomatic single-level degenerative anterolisthesis and stenosis without scoliosis underwent decompression and single-level Dynesys stabilisation at the level of degenerative anterolisthesis. Patients were followed prospectively for 24 months with radiographs, Oswestry Disability Index scores, visual analogue scale (VAS) for back and leg pain, and estimated pain-free walking distance.
\end{abstract}

\footnotetext{
M. Payer

Neurosurgical Department, Hirslanden Klinik, Zürich, Switzerland

M. Payer $\cdot$ E. Tessitore

Neurosurgical Department, University of Geneva, Geneva,

Switzerland

S. Nicolas

Department of Surgery, Frankston Hospital, Frankston, Victoria, Australia

N. Oezkan

Neurosurgical Department, Universitätsklinikum Essen, Essen, Germany

M. Payer $(\bowtie)$

Klinik Hirslanden, Witellikerstrasse 40, 8032 Zürich, Switzerland

e-mail: mpayer@hotmail.com
}

Results At the 2-year follow-up, back pain was reduced from 6.5 preoperatively to 2.5 , leg pain from 5.4 to 0.6 . The painfree walking distance was estimated at $500 \mathrm{~m}$ preoperatively and at over $2 \mathrm{~km}$ after 2 years, while the ODI decreased from $54 \%$ to $18 \%$. Screw loosening was found in $2 / 30$ cases. Symptomatic adjacent segment disease was found in $3 / 30$ patients between 12 and 24 months postoperatively.

Conclusions Single-level Dynesys stabilisation combined with single- or multi-level decompression seems to be a safe and efficient treatment option in single-level degenerative anterolisthesis and stenosis over an observation period of 2 years, avoiding iliac crest or local bone grafting required by fusion procedures. However, it does not seem to avoid adjacent segment disease.

Keywords Lumbar stenosis - Degenerative spondylolisthesis · Transpedicular stabilisation · Dynamic stabilisation

\section{Introduction}

Symptomatic degenerative lumbar spondylolisthesis with spinal stenosis is one of the most frequent indications for spine surgery, particularly in the ageing adult population, with predominance in postmenopausal women and at the level L4/5 [21]. While decompression alone for symptomatic degenerative lumbar anterolisthesis has shown satisfying results in several series [6, 15, 24, 27, 29], proponents of concomitant fixation argue that decompression can be more reliably achieved and maintained at long term by adding transpedicular stabilisation [1, 4, 16, 18, 23, 28, 33]. Bilateral decompression has also been shown to aggravate symptoms related to instability [13], and long-term results were commonly better after transpedicular stabilisation procedures [2, $19,23]$. Bilateral decompression via a unilateral approach has also been advocated in case of degenerative anterolisthesis 
and stenosis, in order to reduce the risk for postoperative instability [29]. In the past 2 decades, new dynamic transpedicular fixation techniques have gained some acceptance for stabilisation of degenerative lumbar anterolisthesis $[7,14,21,22,30,34,35]$. The aim of this study was to evaluate the safety and efficacy of dynamic transpedicular stabilisation with the Dynesys ${ }^{\circledR}$ system and decompression in single-level degenerative anterolisthesis with symptomatic degenerative stenosis.

\section{Material and methods}

Thirty-three consecutive patients presented with symptomatic single-level degenerative anterolisthesis, all of which were Meyerding grade I, and single- or multi-level stenosis between 2005 and 2009. During the prospective observation period of 2 years, there were three drop-outs: one from unrelated death due to a myocardial infarction, one from new onset of psychiatric disease and one from new onset of cancer. Thus, complete follow-up was achieved in 30 patients (age 46-88, mean 66; 8 men and 22 women). There was no control group. Previous lumbar spine surgery, known osteoporosis, scoliosis beyond a Cobb angle of $20^{\circ}$, multi-level anterolisthesis, spinal infection or tumour and psychiatric disease were exclusion criteria. Patients were operated by two surgeons (M.P. or E.T.) after obtaining informed consent.

\section{Surgical technique}

After induction of general anaesthesia, the patient was placed in the prone position. Through a midline subperiosteal muscle-stripping approach the transverse processes of the anterolisthesis level were exposed on both sides. Dynesys titanium screws (Dynesys; Zimmer, Winterthur, Switzerland) with a diameter of $6.4 \mathrm{~mm}$ and a length of $40-50 \mathrm{~mm}$, according to individual anatomy, were inserted into the pedicles under lateral and AP fluoroscopic guidance. Polymer cords, which act as tension bands limiting flexion, were inserted to connect the pedicle screws. Polycarbonate urethane spacers, which limit extension by resisting compressive loads, were cut 1-2 mm longer than the measured distance between the pedicle screw heads and slid over the cords. Finally the cord was pulled tight to a pre-calibrated tension and locked on the pedicle screw heads, assuring calibrated and optimal screwspacer force and bone-screw stress (Fig. 1). Midlinepreserving micro-decompression up to the medial pedicle walls was then performed bilaterally on the anterolisthesis level and, if relevant stenosis was present, on the adjacent level(s). Patients were mobilised from the first postoperative day on with an elastic brace for 6 weeks.

The patients were followed prospectively for 24 months with plain standing antero-posterior and lateral radiographs,

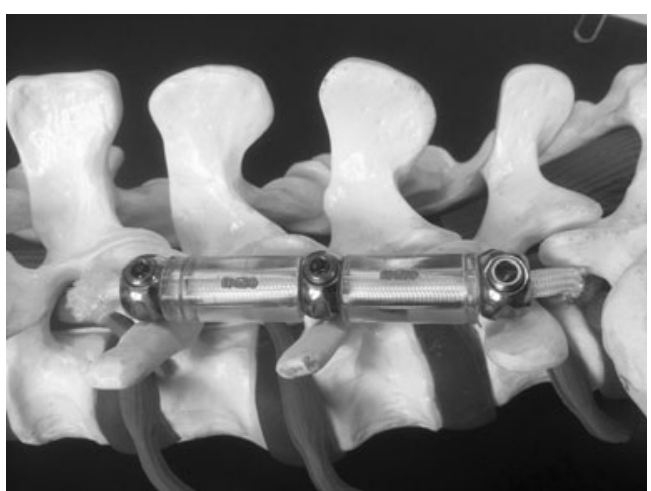

Fig. 1 Postero-lateral view of a two-level Dynesys sawbone model

Oswestry Disability Index scores (ODI), visual analogue scale (VAS) for back and leg pain, and estimated pain-free walking distance.

Statistical methods

Continuous variables are summarised as medians and interquartile ranges (IQR). Paired before and after visual analogue scores (VAS) are compared using the sign-rank test. Categorical data is presented as frequencies and percents.

Clinically significant change is considered the minimal amount of change on the Oswestry Disability Index (ODI) that is required to suggest that a clinically relevant positive change has occurred. This is a more relevant method than using probability tests ( $p$ values) to define change, because clinically significant change is more important to clinical practice than statistically significant change. Clinically significant change has been defined elsewhere, and is considered to be a change of 12.8 points on the ODI [5]. Thus those with a change greater than 12.8 are considered to be "responders", and those with changes less than 12.8 are considered to be "non-responders".

\section{Results}

The results are summarised in Table 1. The median age was 66 years of age, with an interquartile range (IQR) of 15 years. Median operative time was $150 \mathrm{~min}$ (IQR $40 \mathrm{~min}$ ). Median length of stay was 9 days, (IQR 3 days). Baseline Oswestry Disability Index was 53 (IQR 1) for this patient cohort. Stabilisation was performed at L3/4 in 3 patients, at L4/5 in 25 patients, and at L5/S1 in 2 patients. Decompression was performed only at the anterolisthesis level (single-level decompression) in 16 cases, at the anterolisthesis and one adjacent level (double-level decompression) in 9 cases, and at the anterolisthesis and two adjacent levels (three-level decompression) in 5 cases. 
Table 1 Clinical and radiological results

\begin{tabular}{|c|c|c|c|c|}
\hline & Baseline & 6 months & 12 months & 24 months \\
\hline Low-back VAS & $6(3)$ & $2(2)^{*}$ & $2(2)^{* *}$ & $3(3)^{* *}$ \\
\hline Left-leg VAS & $6(5)$ & $0(1)^{*}$ & $0(1)^{* *}$ & $0(1)^{* *}$ \\
\hline Right-leg VAS & $6.5(5)$ & $0(1)^{*}$ & $0(1)^{* *}$ & $0(1)^{* *}$ \\
\hline Oswestry Disability Index change, $n(\%)$ & - & $27(92 \%)$ & $27(92 \%)$ & $27(92 \%)$ \\
\hline Screw misplacement (X-rays) & - & $0(0 \%)$ & $0(0 \%)$ & $0(0 \%)$ \\
\hline Screw loosening (X-rays) & - & $1(3 \%)$ & $2(7 \%)$ & $2(7 \%)$ \\
\hline Symptomatic ASD & - & $0(0 \%)$ & $0(0 \%)$ & $3(10 \%)$ \\
\hline
\end{tabular}

Medians (IQR) or frequency (\%)

Each time-point is compared with the previous time-point using the sign-rank test. For example, the 6-month VAS is compared with the baseline VAS and the 12-month VAS is compared with the 6-month VAS

$A S D$ adjacent segment disease, $V A S$ visual analogue scale

$* p$ value $<0.001,{ }^{* *} p$ value $>0.1$

Four complications occurred: one dural breach that could be sutured, one deep venous thrombosis during the hospital stay, treated by anticoagulation, and two urinary infections during hospital stay, treated with oral antibiotics.

\section{Clinical outcomes}

Low-back pain on a visual analogue scale (VAS) improved from a median of 6 preoperatively (IQR 3) to 2 (IQR 2 ) at 6 months (sign-rank test; $p<0.001$ ), 2 (IQR 2 ) at 12 months, and 3 (IQR 3 ) at 24 months. The VAS for low-back pain did not change after 6 months (sign-rank test; $p>0.09$ at 12 and 24 months).

Radicular pain, down the left leg on a VAS improved from a median of 6 preoperatively (IQR 5) to 0 (IQR 1 ) at 6 months, 0 (IQR 1 ) at 12 months, and 0 (IQR 1 ) at 24 months. The VAS for radicular pain in the left leg did not change after 6 months $(p>0.10)$.

Radicular pain, down the right leg on a VAS improved from a median of 6.5 preoperatively (IQR 5) to 0 (IQR 1 ) at 6 months, 0 (IQR 1) at 12 months, and 0 (IQR 1) at 24 months. The VAS for radicular pain in the left leg did not change after 6 months $(p>0.10)$. The mean estimated walking distance improved from $<500 \mathrm{~m}$ to $>2,000 \mathrm{~m}$ at 6 , 12, 24 months.

The mean Oswestry Disability Index (0-100 \% scale) decreased from $54 \%$ preoperatively to $17 \%$ at 6 months, $14 \%$ at 12 months, and $18 \%$ at 24 months. As defined by clinically significant change, 27 (92\%) patients were found to have responded positively to surgery at the 6 month follow-up period based on the Oswestry Disability Index. This figure remained the same at 12 and 24 months.

Return to work concerned only a minority of patients, as the majority was in retirement age. Nine out of 30 patients had full-time professional employment before surgery ; full-time and half-time activity was regained by four and five patients respectively at 6 months, by six and three patients at 12 months, and by six and two patients at 24 months, by which time one patient had retired.

\section{Radiological results}

No obvious screw misplacement on plain X-rays was observed over the observation period according to the authors' analysis and the radiology reports (Fig. 2). Screw loosening was found on plain radiographs in $2 / 30$ patients. One was a 57 -year-old woman with obesity and non-insulin-dependent diabetes; VAS for back pain was 5 at the 2-year follow-up without pain medication, but the patient refused a computed tomography (CT) scan as re-operation was not wanted. The other one was a 78-year-old woman, who presented with asymptomatic screw loosening on X-ray control at 1- and 2year follow-up; surgical revision was not needed.

Symptomatic adjacent segment degeneration was observed in $3 / 30$ patients $(10 \%)$. One patient presented with suprajacent stenosis at L3/4 at 18 months after decompression and Dynesys stabilisation at L4/5; after initial response to facet blocks, decompression and Dynesys extension to the L3/4 level was performed 3 years (hence after the study period) after the index surgery. Another patient started to suffer from symptomatic subjacent foraminal stenosis at L5/S1 1 year after L2/5 decompression and L4/5 Dynesys stabilisation and had to be re-operated by extraforaminal microdecompression 14 months after the index surgery. The third patient developed symptomatic disc protrusion and mild stenosis two levels above the initial L5/S1 decompression and Dynesys stabilisation; conservative therapy with steroid injection at the new level resolved the symptoms. Altogether, there was one re-operation within the 2-year follow-up period. 
a

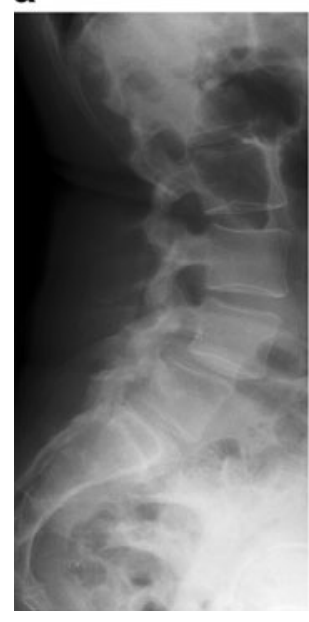

b

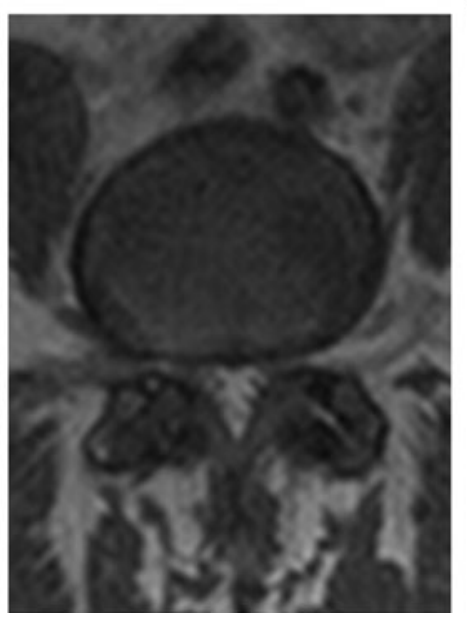

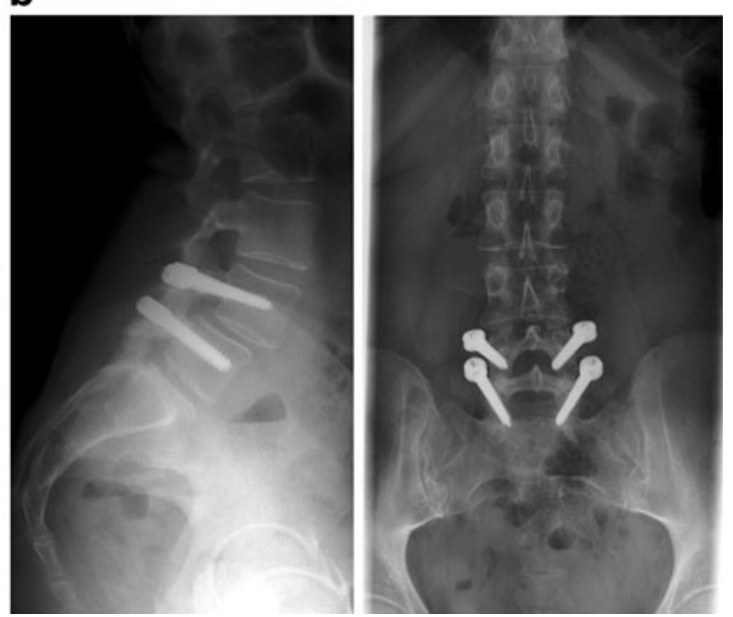

Fig. 2 A 49-year-old woman with symptomatic stenosis and degenerative anterolisthesis at L4/5. a Preoperative standing lateral radiograph and axial T2 MRI at L4/5. b Standing lateral and AP radiographs at 24 months after decompression and dynamic pedicle fixation

\section{Discussion}

Approximately $92 \%$ of our patients were found to have responded positively to surgery, or, in other words, achieved a positive clinically meaningful change at the 6-month followup (no $p$ value required, as this was a clinically meaningful change). It appears that the benefits achieved from surgery reach their maximum at the 6-month time-point. Thus, it is reasonable to counsel patients that any further benefit from surgery is unlikely to occur once they are 6 months postsurgery.

There are several hypothetical advantages of dynamic pedicle stabilisation over instrumented fusion for degenerative lumbar instability. First, there is no need for bone grafting and patients are not exposed to potential donor site complications, such as long-term donor site pain, haematoma from bone bleeding or even pelvic fracture through the donor site. Second, no bone substitutes with potential infectious disease transmission and reduced fusion properties have to be applied $[30,34,35]$. Finally, there is no need for cage insertion and its potential complications such as excessive root retraction, cage migration or subsidence [35]. Avoiding fusion altogether simplifies the surgical procedure and shortens its duration [30, 34, 35]. However, such theoretical superiority of the Dynesys device is counterbalanced by its potential downsides, such as ongoing pain from incomplete immobilisation, lack of anterior column support and a higher rate of screw loosening [11, 17, 23]. Its actual usefulness has therefore only gained controversial acceptance in the spine community.

Biomechanically, the Dynesys system has been shown to be only little dynamic $[8,12,25,31,32]$. A preclinical investigation on ten cadaveric lumbar spine segments (L2L5) showed that implantation of Dynesys with standard length spacers significantly reduced range of motion (ROM) compared with the intact spine, with the least significant changes seen in axial rotation [25]. Equally, a subsequent study on 21 fresh-frozen human lumbar spine motion segments revealed a ROM reduction of $68 \%$ in flexionextension, $68 \%$ in lateral bending, and only $13 \%$ in axial rotation after decompression and Dynesys implantation compared with the intact spine. The study confirms that implantation of the Dynesys device after decompression leads to a restriction of ROM in all motion planes, whereby axial rotation is only mildly affected [32].

We identified three clinical English language publications about Dynesys instrumentation focusing on the specific indication of lumbar degenerative spondylolisthesis [11, 30, 34]. Hoppe et al. [11] recently reported 39 consecutive patients with symptomatic degenerative lumbar spondylolisthesis at L4/5, treated with bilateral decompression and Dynesys instrumentation. At a mean follow-up of 7.2 years back pain improved in $89 \%$ and leg pain improved in $86 \%$ of patients. Symptomatic adjacent segment disease was found in 6/39 cases, asymptomatic screw loosening in $4 / 39$ cases, late onset infection in one case and screw breakage in one case. The authors concluded that Dynesys stabilisation is a valid alternative to other stabilisation devices in symptomatic L $4 / 5$ degenerative spondylolisthesis.

Schaeren et al. [30] investigated 19 consecutive patients, who underwent decompression and Dynesys stabilisation for symptomatic lumbar stenosis and degenerative spondylolisthesis. Patients were evaluated clinically and radiologically after a minimum follow-up of 4 years with a mean follow-up of 52 months. Pain on VAS and walking distance improved significantly at 2 years and remained unchanged at 4-year follow-up; 3/19 patients showed slight screw-loosening at 2- and 4year follow-ups and 1/19 patients had screw breakage. 
a

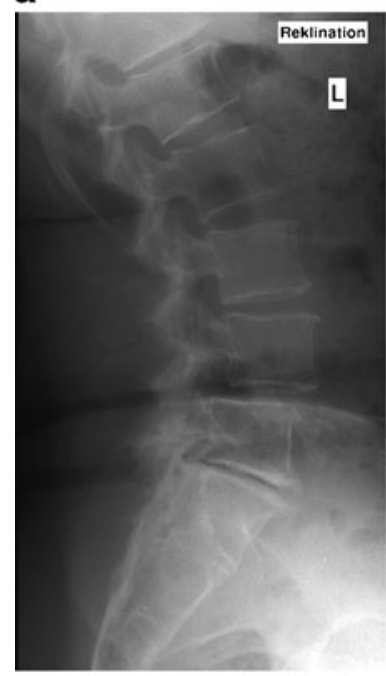

C

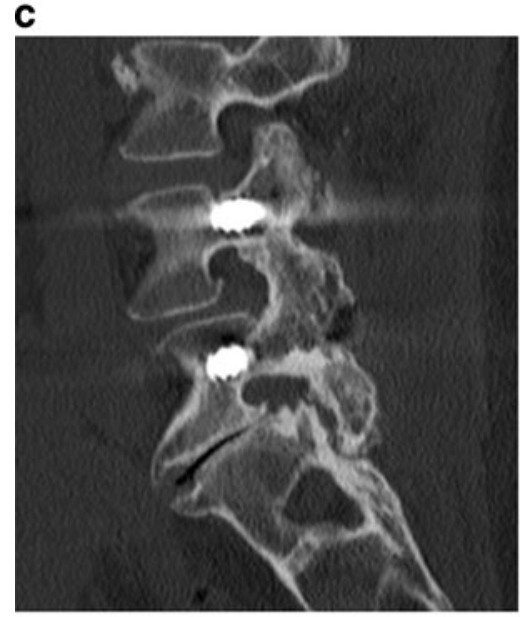

b

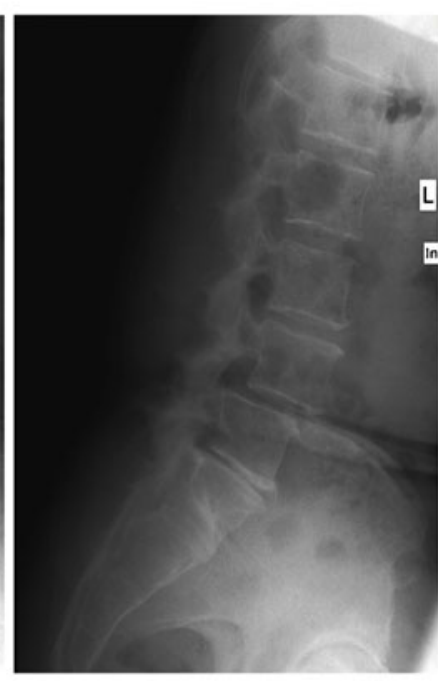

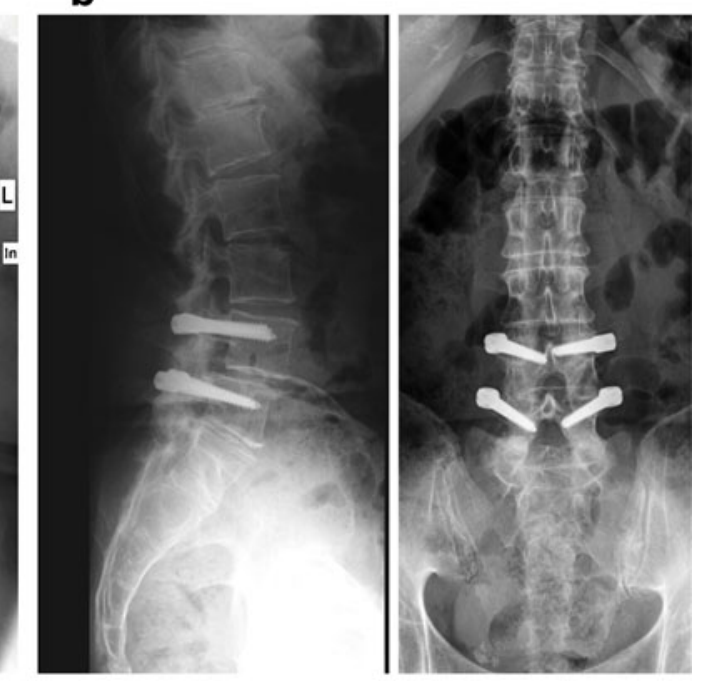
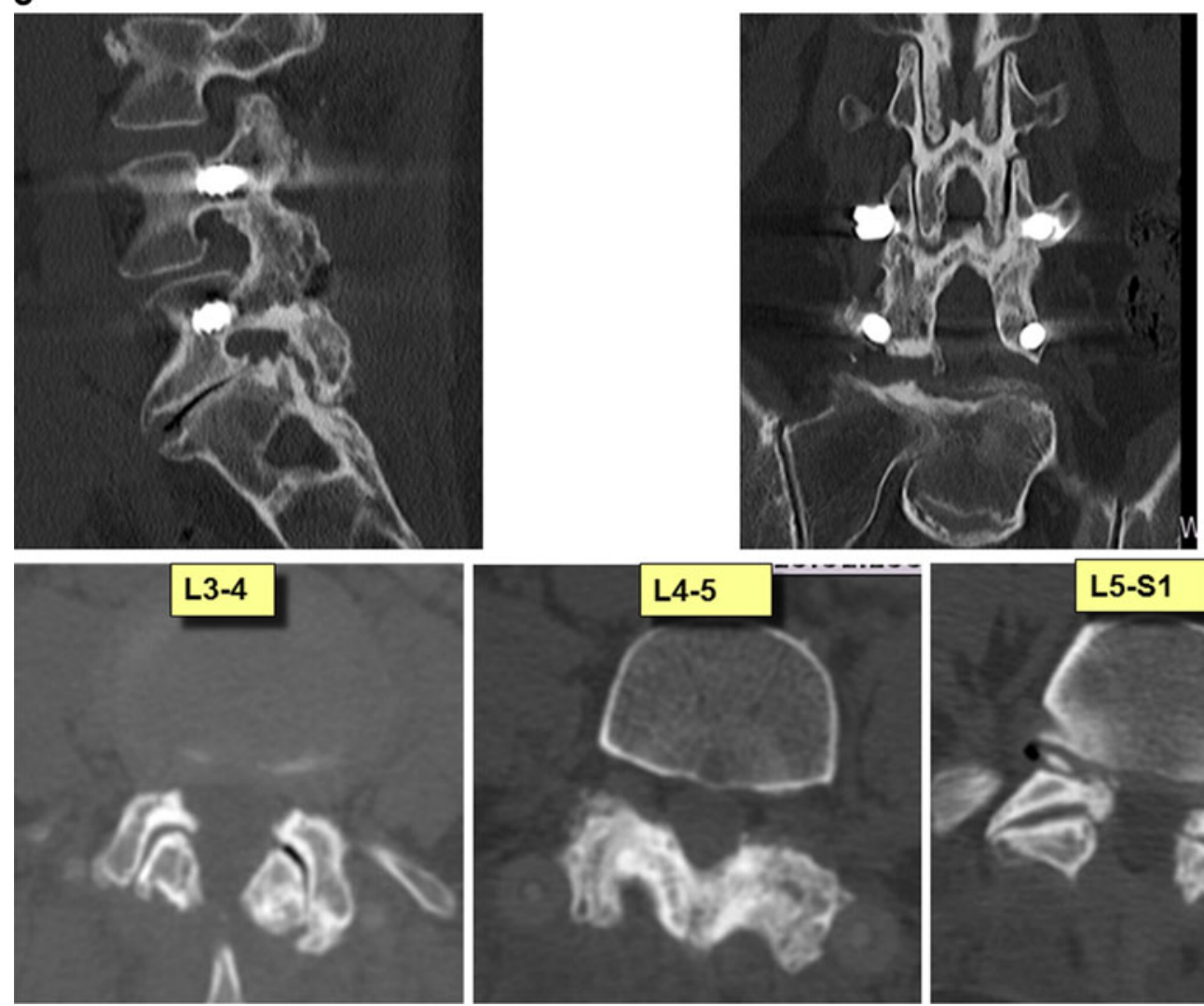

Fig. 3 A 64-year-old woman with symptomatic stenosis and degenerative anterolisthesis at L4/5, who developed symptomatic subjacent foraminal stenosis at L5/S1 within 14 months after decompression and dynamic stabilisation at L4/5. a Preoperative lateral radiographs in

At 4-year follow-up, $47 \%$ of the patients showed some degeneration at adjacent levels, with a patient satisfaction of $95 \%$.

The authors conclude that in elderly patients with spinal stenosis and degenerative spondylolisthesis, decompression and dynamic stabilisation leads to excellent clinical and flexion-extension. b Standing lateral and AP radiographs at 14 months after decompression and dynamic pedicle fixation. c Axial CT cuts and sagittal and coronary reconstruction, revealing facet joint ankylosis at 14 months postoperatively

radiological results, maintaining enough stability to prevent progression of spondylolisthesis.

Welch et al. [34], in a multicentre randomised prospective trial, investigated 101 patients from six sites, who underwent Dynesys stabilisation of the lumbar spine in the presence of one- or two-level degenerative spondylolisthesis or 
retrolisthesis and stenosis. The mean pain and function scores improved significantly from the baseline to the 12-month follow-up evaluation. The authors conclude that Dynesys may be preferable to fusion for surgical treatment of degenerative spondylolisthesis and stenosis because it decreases back and leg pain, while it avoids the morbidity of donor site problems encountered in fusion.

Our clinical results with significant improvement of back and leg pain over 24 months observation time, symptomatic adjacent segment disease in 3/30 cases, and obvious screw loosening in 2/30 cases are consistent with the findings of Hobbe et al., Schaeren et al. and Welch et al. Symptomatic adjacent segment disease after mono-segmental Dynesys stabilisation seems to lie within the range of fusions and not below [26].

Several other studies have reported single- or multi-level use of Dynesys for various indications, including previously operated patients, spondylolisthesis, osteochondrosis, spondylarthrosis, recurrent disc herniation, or simply stabilising lumbar decompression even in the absence of instability $[3,9,10,16]$. However, evaluation of such heterogeneous cohorts with larger patient numbers makes interpretation meaningless and does not lead to conclusions for Dynesys indications.

So far, no clinical study has shown its long-term motion preservation. Fayyazi et al. [7] placed three to five tantalum beads in each vertebral body of six patients, who underwent posterior decompression followed by posterior stabilisation using Dynesys instrumentation (4 one-level, 2 two-levels). Postoperative radiographs in flexion, extension, right, and left lateral bending, and three-dimensional reconstruction through radiostereometric analysis at 3, 6, 12, and 24 months postoperatively revealed that the segmental motion of the implanted level was only minor and considerably less than normal spinal motion. Facet joint ankylosis may occur over time: all three patients in our current study, in whom a CT was performed during the follow-up period, showed facet joint ankylosis within 2 years postoperatively (Fig. 3). This does not mean that all Dynesys stabilisations must result in ankylosis eventually, as segmental motion depends on the amount of applied distraction, disc height, patient's age and facet joint arthrosis. However, even if ankylosis occurs gradually, neighbour segments may have less initial mechanical stress from a dynamic than a traditional rigid pedicle fixation. Furthermore, disc degeneration at the bridged and adjacent segment has been shown to continue within 2 years after Dynesys stabilisation [20].

Screw loosening in dynamic pedicle fixation has been a concern as in rigid fixation techniques. Focusing on Dynesys use in single-level degenerative anterolisthesis, a loosening rate in $10.3 \%$ of patients or $4 / 39$ [11] and $15.8 \%$ or $3 / 19$ [30] has been reported. Even though this loosening rate for singlelevel dynamic stabilisation seems rather high, the overall patient numbers are small and screw loosening was described as asymptomatic in both series. Ko et al. [17], in their study on Dynesys screw loosening in 71 patients with Dynesys stabilisation for one- or two-level lumbar spondylosis, found radiographic evidence of screw loosening in $19.7 \%$ or $14 / 71$ of patients within a mean follow-up of 17 months. Nonetheless, loosening of screws had no adverse clinical effect in that series. Only larger series with systematic CT follow-up would yield a more accurate rate of screw loosening and its potential clinical impact.

Altogether, decompression and dynamic transpedicular stabilisation with the Dynesys system seems to lead to good results in symptomatic degenerative anterolisthesis with stenosis over a 2-year observation period. As this study exclusively focused on single-level degenerative anterolisthesis, no conclusion can be drawn for the use of the Dynesys system in any other indications.

The current study has several limitations: lack of a control group, limited case number and lack of systematic CT analysis. However, the latter would have exposed many patients to unnecessary radiation and caused additional costs. On the other hand, this study analysed the Dynesys system in a clearly defined single-level indication, through a uniform surgical technique, and documented prospectively over 2 years.

\section{Conclusions}

On the basis of the current study, single-level Dynesys stabilisation and decompression in patients with symptomatic degenerative anterolisthesis with stenosis seems safe and efficient over a 2-year observation period, with $92 \%$ of patients achieving a positive clinically significant change (good result). The results are within the range of traditional transpedicular fusion and decompression reports for the same indication. No need for bone grafting or bone substitutes, no need for cage insertion and preservation of motion at least temporarily are hypothetical advantages of the Dynesys stabilisation. However, single-level Dynesys stabilisation does not seem to avoid adjacent segment disease.

\section{Conflicts of interest None.}

Disclaimer The authors declare that they did not and will not receive any benefits from the implant company (Zimmer) or any other party for this study.

\section{References}

1. Abdu WA, Lurie JD, Spratt KF, Tosteson AN, Zhao W, Tosteson TD, Herkowitz H, Longely M, Boden SD, Emery S, Weinstein JN (2009) Degenerative spondylolisthesis: does fusion method influence outcome? Four-year results of the spine patient outcomes research trial. Spine 34(21):2351-2360 
2. Booth KC, Bridwell KH, Eisenberg BA, Baldus CR, Lenke LG (1999) Minimum5-year results of degenerative spondylolisthesis treated with decompression and instrumented posterior fusion. Spine 24:1721-1727

3. Bothmann M, Kast E, Boldt GJ, Oberle J (2008) Dynesys fixation for lumbar spine degeneration. Neurosurg Rev 31(2):189-196

4. Bridwell KH, Sedgewick TA, O'Brien MF, Lenke LG, Baldus C (1993) The role of fusion and instrumentation in the treatment of degenerative spondylolisthesis with spinal stenosis. J Spinal Disord 6(6):461-472

5. Copay AG, Glassman SD, Subach BR, Berven S, Schuler TC, Carreon LY (2008) Minimum clinically important difference in lumbar spine surgery patients: a choice of methods using the Oswestry Disability Index, Medical Outcomes Study questionnaire Short Form 36, and pain scales. Spine J 8(6):968-974

6. Epstein NE (1998) Decompression in the surgical management of degenerative spondylolisthesis: advantages of a conservative approach in 290 patients. J Spinal Disord 11(2):116-122

7. Fayyazi AH, Ordway NR, Park SA, Fredrickson BE, Yonemura K, Yuan HA (2010) Radiostereometric analysis of postoperative motion after application of dynesys dynamic posterior stabilization system for treatment of degenerative spondylolisthesis. J Spinal Disord Tech 23(4):236-241

8. Gédet P, Haschtmann D, Thistlethwaite PA, Ferguson SJ (2009) Comparative biomechanical investigation of a modular dynamic lumbar stabilization system and the Dynesys system. Eur Spine J 18(10):1504-1511

9. Grob D, Benini A, Junge A, Mannion AF (2005) Clinical experience with the Dynesys semirigid fixation system for the lumbar spine: surgical and patient-oriented outcome in 50 cases after an average of 2 years. Spine 30(3):324-331

10. Haddad B, Makki D, Konan S, Park D, Khan W, Okafor B (2013) Dynesys dynamic stabilization: less good outcome than lumbar fusion at 4-year follow-up. Acta Orthop Belg 79(1):97-103

11. Hoppe S, Schwarzenbach O, Aghayev E, Bonel H, Berlemann U (2012) Long-term outcome after monosegmental L4/5 stabilization for degenerative spondylolisthesis with the dynesys device. J Spinal Disord Tech. doi:10.1097/BSD.0b013e318277ca7a

12. Jahng TA, Kim YE, Moon KY (2013) Comparison of the biomechanical effect of pedicle-based dynamic stabilization: a study using finite element analysis. Spine J 13(1):85-94

13. Jang JW, Park JH, Hyun SJ, Rhim SC (2012) Clinical outcomes and radiologic changes following microsurgical bilateral decompression via a unilateral approach in patients with lumbar canal stenosis and Grade I degenerative spondylolisthesis with a minimum 3-year follow-up. J Spinal Disord Tech 15: doi:10.1097/BSD. ob013e31827566a8

14. Kaner T, Dalbayrak S, Oktenoglu T, Sasani M, Aydin AL, Ozer AF (2010) Comparison of posterior dynamic and posterior rigid transpedicular stabilization with fusion to treat degenerative spondylolisthesis. Orthopedics 33(5)

15. Kinoshita T, Ohki I, Roth KR, Amano K, Moriya H (2001) Results of degenerative spondylolisthesis treated with posterior decompression alone via a new surgical approach. J Neurosurg 95(1 Suppl):11-16

16. Kleinstueck FS, Fekete TF, Mannion AF, Grob D, Porchet F, Mutter U, Jeszenszky D (2012) To fuse or not to fuse in lumbar degenerative spondylolisthesis: do baseline symptoms help provide the answer? Eur Spine J 21(2):268-275

17. Ko CC, Tsai HW, Huang WC, Wu JC, Chen YC, Shih YH, Chen HC, Wu CL, Cheng H (2010) Screw loosening in the Dynesys stabilization system: radiographic evidence and effect on outcomes. Neurosurg Focus 28(6):E10

18. Konno S, Kikuchi S (2000) Prospective study of surgical treatment of degenerative spondylolisthesis: comparison between decompression alone and decompression with graf system stabilization. Spine 25(12):1533-1537

19. Kornblum MB, Fischgrund JS, Herkowitz HN, Abraham DA, Berkower DL, Ditkoff JS (2004) Degenerative lumbar spondylolisthesis with spinal stenosis. A prospective long term study comparing fusion and pseudarthrosis. Spine 29:726-733

20. Kumar A, Beastall J, Hughes J, Karadimas EJ, Nicol M, Smith F, Wardlaw D (2008) Disc changes in the bridged and adjacent segments after Dynesys dynamic stabilization system after two years. Spine 33(26):2909-2914

21. Lawhorne TW 3rd, Girardi FP, Mina CA, Pappou I, Cammisa FP Jr (2009) Treatment of degenerative spondylolisthesis: potential impact of dynamic stabilization based on imaging analysis. Eur Spine J 18(6):815-822

22. Majid K, Fischgrund JS (2008) Degenerative lumbar spondylolisthesis: trends in management. J Am Acad Orthop Surg 16(4):208-215

23. Martin CR, Gruszczynski AT, Braunsurth HA, Fallatah SM, O’Neil J, Wai EK (2007) The surgical management of degenerative lumbar spondylolisthesis: a systematic review. Spine 32:1791-1798

24. Müslüman AM, Cansever T, Yılmaz A, Çavuşoğlu H, Yüce İ, Aydın Y (2012) Midterm outcome after a microsurgical unilateral approach for bilateral decompression of lumbar degenerative spondylolisthesis. J Neurosurg Spine 16(1):68-76

25. Niosi CA, Zhu QA, Wilson DC, Keynan O, Wilson DR, Oxland TR (2006) Biomechanical characterization of the three-dimensional kinematic behaviour of the Dynesys dynamic stabilization system: an in vitro study. Eur Spine J 15(6):913-922

26. Park P, Garton HJ, Gala VC, Hoff JT, McGillicuddy JE (2004) Adjacent segment disease after lumbar or lumbosacral fusion: review of the literature. Spine 29(17):1938-1944

27. Park JH, Hyun SJ, Roh SW, Rhim SC (2012) A comparison of unilateral laminectomy with bilateral decompression and fusion surgery in the treatment of grade I lumbar degenerative spondylolisthesis. Acta Neurochir (Wein) 154(7):1205-1212

28. Rousseau MA, Lazennec JY, Bass EC, Saillant G (2005) Predictors of outcomes after posterior decompression and fusion in degenerative spondylolisthesis. Eur Spine J 14(1):55-60

29. Sasai K, Umeda M, Maruyama T, Wakabayashi E, Iida H (2008) Microsurgical bilateral decompression via a unilateral approach for lumbar spinal canal stenosis including degenerative spondylolisthesis. J Neurosurg Spine 9(6):554-559

30. Schaeren S, Broger I, Jeanneret B (2008) Minimum four-year followup of spinal stenosis with degenerative spondylolisthesis treated with decompression and dynamic stabilization. Spine 33(18):E636-E642

31. Schilling C, Krüger S, Grupp TM, Duda GN, Blömer W, Rohlmann A (2011) The effect of design parameters of dynamic pedicle screw systems on kinematics and load bearing: an in vitro study. Eur Spine J 20(2):297-307

32. Schulte TL, Hurschler C, Haversath M, Liljenqvist U, Bullmann V, Filler TJ, Osada N, Fallenberg EM, Hackenberg L (2008) The effect of dynamic, semi-rigid implants on the range of motion of lumbar motion segments after decompression. Eur Spine J 17(8):1057-1065

33. Sengupta DK, Herkowitz HN (2005) Degenerative spondylolisthesis: review of current trends and controversies. Spine 30(6 Suppl):S71S81

34. Welch WC, Cheng BC, Awad TE, Davis R, Maxwell JH, Delamarter R, Wingate JK, Sherman J, Macenski MM (2007) Clinical outcomes of the Dynesys dynamic neutralization system: 1-year preliminary results. Neurosurg Focus 22(1):E8

35. Yu SW, Yang SC, Ma CH, Wu CH, Yen CY, Tu YK (2012) Comparison of Dynesys posterior stabilization and posterior lumbar interbody fusion for spinal stenosis L4L5. Acta Orthop Belg 78(2): 230-239 